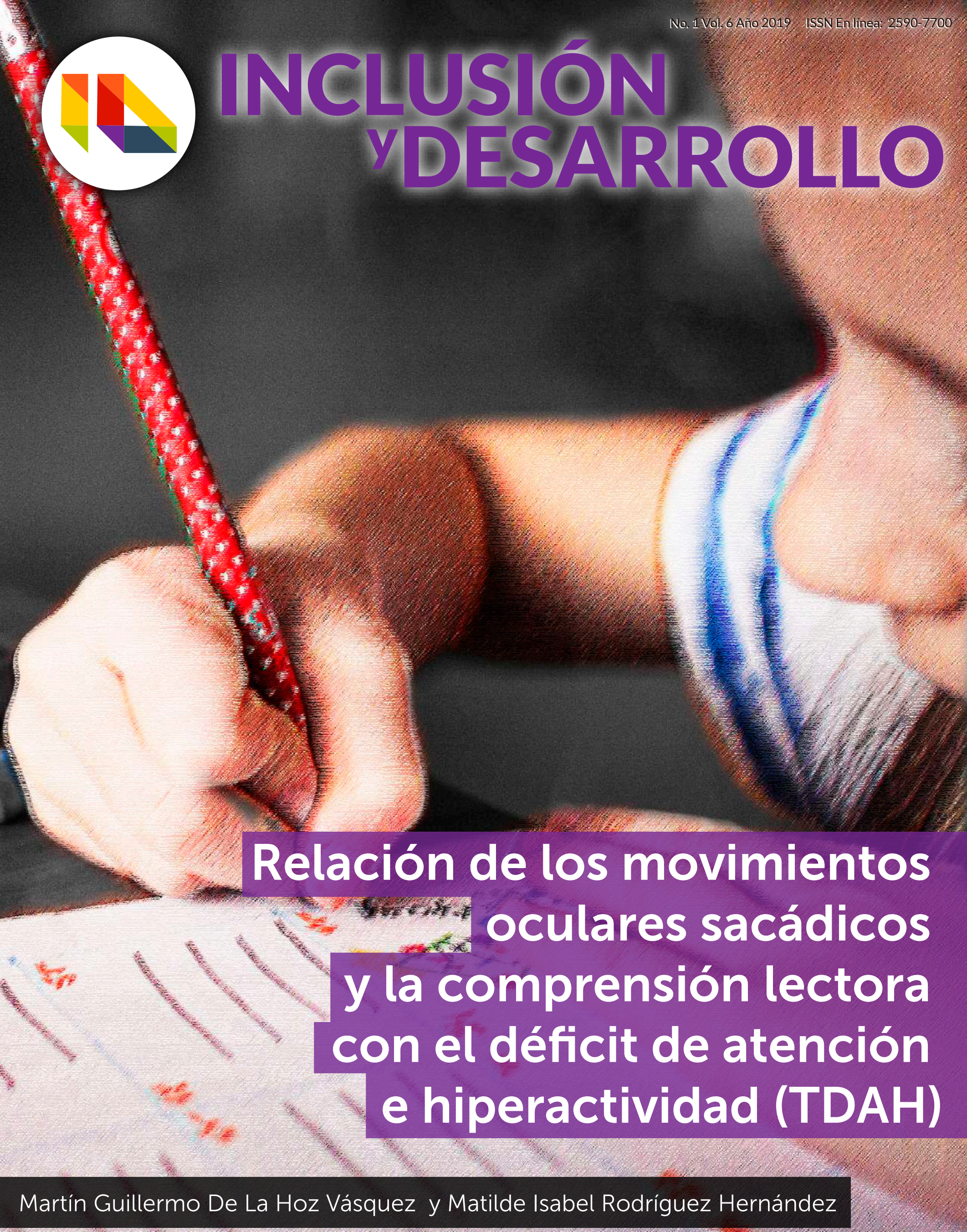




\title{
RELACIÓN DE LOS MOVIMIENTOS OCULARES SACÁDICOS Y LA COMPRENSIÓN LECTORA CON EL DÉFICIT DE ATENCIÓN E HIPERACTIVIDAD (TDAH)
}

\author{
RELATIONSHIP OF SACCADIC EYE MOVEMENTS \\ AND READING COMPREHENSION \\ WITH ATTENTION DEFICIT HYPERACTIVITY DISORDER (ADHD)
}

Martín Guillermo De La Hoz Vásquez martindelahoz@mail.uniatlantico.edu.co Universidad del Atlántico Barranquilla-Colombia

Matilde Isabel Rodríguez Hernández matildeirodriguez@mail.uniatlantico.edu.co Universidad del Atlántico Barranquilla-Colombia

\section{Resumen}

Objetivo. Establecer una posible relación entre el TDAH, la calidad de los movimientos sacádicos y la comprensión lectora en niños de 7 a 12 años con y sin déficit de atención e hiperactividad.

Diseño. Estudio correlacional empleando el método inductivo

Metodología. Participaron. 30 niños, 15 con TDAH y 15 sin el trastorno que cursaban de primero a quinto grado de primaria en la institución educativa distrital Simón Bolívar a los cuales se les aplicó una prueba de comprensión lectora (Prolec-r) y un test de movimientos sacádicos (King Devick).

Resultados. Los resultados obtenidos no relacionan significativamente la baja comprensión lectora con unos movimientos oculares inadecuados

Conclusión. No se evidencian problemas significativos en los movimientos sacádicos y en la comprensión lectora de los niños con TDAH en comparación con el grupo control. Se plantea un programa de intervención para la mejora de la comprensión de lectura y otro para corregir las dificultades presentadas en los movimientos sacádicos.

Palabras clave: TDAH, movimientos sacádicos, comprensión de lectura, prolec- r, King Devick 


\section{Abstract}

Objective. Establish a possible relationship between ADHD, the quality of saccades and reading comprehension in children from 7 to 12 years old with and without this disorder.

Design. A correlation study was performed using the inductive method

Methodology. For which we took a sample of 30 children, 15 with ADHD and 15 without the disorder in grades between first and fifth at Simon Bolivar school, who were tested in reading comprehension (Prolec-r) and saccades (King Devick).

Results. The results we got relate low reading comprehension with inadequate eye movements. Conclusion. There are no significant problems in the saccades and reading comprehension of children with ADHD compared to the control group. We propose an intervention program for improving reading comprehension and another one for correcting the difficulties presented with saccades.

Keywords: TDAH, reading comprehension, saccades, prolec- r, King Deving. 


\section{INTRODUCCIÓN}

$\mathbf{E}_{\mathrm{c}}^{\mathrm{n}}$ la actualidad se encuentra en las aulas de clase un alto porcentaje de niños con déficit de atención e hiperactividad, muchos de ellos carecen de diagnóstico clínico, sin embargo, al presentar las mismas características de niños con TDAH que ya han sido valorados por especialistas, se facilita su detección en el ambiente escolar, debido a que exhiben sus mismos comportamientos y sintomatologías. Estos problemas son dificultades de comprensión que igualmente presentan estudiantes sin este déficit entre un 5 y un $8 \%$. Este porcentaje aumenta en la población de estudiantes con trastorno por déficit de atención e hiperactividad (TDAH) Miranda-Casas, et al (2010 p. 135). Cabe mencionar que las falencias presentadas como son dificultades al leer un texto sin regresiones u omisiones, necesitar más tiempo al realizar las tareas, entre otras, son generalmente más significativas en los niños que presentan TDAH.

La comprensión de lectura en los niños con TDAH ha sido motivo de interés para los investigadores a lo largo del tiempo, estudios realizados han analizado si los niños con el trastorno tienen patologías oculares o si presentan alguna deficiencia visual o viso-perceptual por la que desarrollen una atención y concentración inadecuadas Farrar et al (2001), Granet et al ( 2005) y Borsting et al (2005) otros estudios realizados en los últimos años establecen que existe relación entre el trastorno específico del aprendizaje (TEA) y el Trastorno por déficit de atención e hiperactividad (TDAH) entre estas investigaciones podemos citar la realizada por Varela \& Mejía (2014), en la ciudad de Manizales en la cual se evaluó a 60 niños en edades comprendidas entre los siete y once años evidenciándose en estos una alta comorbilidad del TDAH con el TEA. Otros investigadores han abordado diferentes temáticas referentes a los niños con TDAH algunos de ellos se han interesado en las estrategias que utilizan los docentes en el aula para mejorar la comprensión lectora entre ellos Alonso (2016) quien en su tesis doctoral evalúa como después de instruir a los niños con TDAH en el reconocimiento de las estructura de los diferentes tipos de textos obtienen mejores resultados en la comprensión de lectura, mientras que otros autores hacen énfasis en sus trabajos en los niveles de comprensión lectora, producción de textos, lenguaje y comunicación que presentan estos infantes como el presentado por Inostroza (2017) el cual tuvo como resultado que existe una diferencia estadísticamente significativa en el desempeño de los niños con TDAH y los que no presentan el déficit, en investigaciones más recientes se viene estudiando si las funciones ejecutivas influyen sobre la comprensión lectora González et al (2018).

En muchas ocasiones la atención y concentración inapropiadas es causa de un bajo rendimiento académico y fracaso escolar que se evidencia en los problemas de lectura esto se reafirma por lo expuesto en la última versión del Manual Diagnóstico y Estadístico de los Trastornos Mentales (Diagnostic and Statistical Manual of Mental Disorders) DSM-V (2013 p. 67). En dicho manual se contempla como uno de los aspectos específicos que influyen en el rendimiento escolar de los afectados por TDAH es la dificultad en la lectura que afecta a la corrección, la fluidez, velocidad y/o comprensión, por lo que se puede considerar entonces que una de las actividades académicas en las que presentan mayor dificultad los individuos con el trastorno es la comprensión.

Esta investigación expone la relación que tiene el TDAH con variables como movimientos sacádicos y comprensión lectora y como estas pueden influir en el rendimiento escolar de los niños afectados con este trastorno. Debido a que no existe documentación suficiente de la relación que existe entre el TDAH y los movimientos sacádicos que afectan a la comprensión lectora es necesario indagar acerca de este tema y profundizar en la percepción visual de los niños diagnosticados con este trastorno y de aquellos que no presentan este déficit por lo cual esta investigación es pertinente.

El objetivo principal de la investigación, fue establecer una posible relación entre el TDAH, la calidad de los movimientos sacádicos y la comprensión lectora en niños de 7 a 12 años con y sin déficit de atención e hiperactividad. Se buscó conocer las diferencias que existen en los movimientos sacádicos evaluando las tareas antisacádicas, prosacádicas y de fijación de los niños que conforman el grupo experimental y del grupo control. Se analizaron las diferencias y se observó cómo se afecta el nivel de comprensión lectora cuando existe una dificultad en los movimientos oculares. Se tomó una muestra conformada por 
30 niños de edades comprendidas entre 7 y 12 años con el fin de evaluar los movimientos oculares de los dos grupos con las pruebas King Deving (K-D) y de comprensión lectora (Prolec-r).

\section{MARCo TEÓRICO}

\section{Comprensión lectora}

La comprensión de un texto es un proceso altamente complejo debido a que es necesario interpretar utilizando la decodificación fonológica y el reconocimiento de palabras, sin embargo, comprender un texto es mucho más que esto debido que implica desarrollar diversos procesos cognitivos-lingüísticos que son decisivos en el proceso de comprensión lectora y que se encuentran afectados en niños con TDAH tales como atención, memoria, abstracción, análisis, síntesis, inferencia, predicción, comparación entre otros.

Autores como Brock y Knapp (1996), Miranda, García y Soriano (2005), Berthiaume, Lorch y Milich (2009) reafirman lo antes expuesto al expresar que los niños afectados con este trastorno presentan algunas dificultades al momento de identificar ideas principales, realizar inferencias y reconocer las incoherencias de un texto. Al reflexionar sobre lo antes señalado se puede decir que los problemas de comprensión lectora son consecuencias de falencias a un nivel primario de decodificación y reconocimiento de palabras debido a que los niños con TDAH omiten palabras o letras y sustituyen unas letras por otras interpretando mal el contenido de la lectura resultado esto igualmente de la impulsividad y de tener afectados los procesos cognitivos-lingüísticos lo cual les impide realizar de manera eficiente y eficaz tareas de atención, comparación, ordenación, análisis etc.

Señalando otros autores cabe mencionar a Cassany (2006) quien equipara el término leer a comprender ya que estima preciso que al leer se realicen procesos cognitivos tales como la anticipación, el aporte de los conocimientos previos, la realización y verificación de hipótesis, así como la elaboración de inferencias, por otro lado Álvarez y González (1996) entienden la comprensión lectora como "el proceso por el que se captan las ideas más importantes del texto, se establecen relaciones y se consigue ejemplificarlas y aplicarlas a diferentes contextos" dicho de otro modo es preciso que el lector capte el sentido del texto y extraiga lo más relevante de este siendo capaz de relacionarlo con la realidad para lo cual debe interactuar con lo escrito así como sugiere Solé (2008), para quien leer es un proceso que requiere que el lector interactúe con el texto para obtener información para los fines que guían la lectura.

La comprensión lectora en niños con TDAH ha sido estudiada por diferentes autores a través del tiempo en diferentes entornos y desde diferentes enfoques coincidiendo muchos autores como los antes mencionados en que los procesos mentales influyen de manera fundamental en la comprensión lectora de igual forma muchos autores hacen énfasis en la importancia de relacionar los saberes previos con el contenido del texto y utilizar estrategias para comprender el texto al tiempo que lo lee, entendiéndose entonces que son tres los factores que determinan que exista una comprensión: el lector, el texto y el contexto.

\section{MOVIMIENTOS SACÁDICOS}

Leer es un proceso que inicia con una etapa sensorial en la que se percibe, fundamentalmente a través de los ojos, la información que se presenta, esta etapa es una de las primordiales ya que si el sistema visual se encuentra afectado no se logrará obtener una correcta decodificación de los signos gráficos y por tanto no se llevará a cabo la comprensión del texto, Según Blázquez, Paul y muñoz (2004), los movimientos oculares tratan de controlar la dirección de los ojos en el momento de percibir objetos en el espacio. El movimiento de los ojos igualmente es importante debido a que el lector que no presenta problemas en una sola fijación puede llevar a cabo el reconocimiento de varias palabras llevando al tiempo una velocidad lectora adecuada y realizando el proceso de comprensión de forma eficaz, a este respecto García-Castellón (2014) expresa que los movimientos oculares son los encargados de que la imagen llegue al ojo y caiga en la retina central durante la lectura.

Aunque es imprescindible que la vista realice la función de forma eficiente y eficaz es el cerebro el que permite comprender lo que se lee según (Martínez Álvarez, 2014), esto lo reafirma (Fajardo, 1999) al exponer que es en el área de Wernicke en donde se realiza la comprensión visual y auditiva, sin embargo no se puede desconocer la función que cumplen los movimientos 
oculares ya que son estos los que intervienen en los primeros niveles de comprensión de la lectura.

Al hablar de Movimientos Oculomotores (MOM) hay que referirnos a la firmeza de fijación, a los movimientos sacádicos y los movimientos de seguimiento. (Scheiman, 1994) expresa que la firmeza de fijación es la capacidad de los músculos para mantener fija la fóvea sobre un objeto y poderlo ver con claridad, sin embargo, hay que aclarar que durante la fijación los ojos no permanecen totalmente inmóviles debido a que realiza movimientos involuntarios logrando mantener fija y nítida la palabra impresa, por otra parte (Ciufreda, 1990) define el movimiento de seguimiento como la capacidad muscular de mantener la fóvea en un objeto que se encuentre en movimiento, son lentos, largos y continuos, en la lectura son los encargados del cambio de renglón, por último los movimientos Sacádicos son aquellos movimientos muy cortos y rápidos (20 a 45 mseg.) usados en el cambio de fijación de un objeto determinado a uno nuevo que despierta su interés. Es el encargado de llevar el ojo a fijar palabra por palabra (espacio de caracteres) en la lectura. Entre menos sacadas se realicen y más se utilice la visión parafoveal, más rápida es la lectura y mejor es su comprensión (Scheiman, 1994). Se puede decir por tanto que la motilidad ocular juega un papel imprescindible en la lectura porque los ojos realizan los movimientos sacádicos y las fijaciones oculares necesarias para leer, reconocer las palabras globalmente o cambiar de renglón, si no se tiene una buena funcionalidad visual esto afecta a la lectura y por consiguiente a la comprensión del texto.

\section{TDAH}

El trastorno por déficit de atención con o sin hiperactividad (TDAH) es el trastorno del neurodesarrollo más frecuente en la infancia Lora-Espinosa y Díaz-Aguilar (2012) El concepto más moderno de TDAH lo ubica como conjuntos complejos y polifacéticos de alteraciones dimensionales de las funciones cerebrales de control cognoscitivo y conductual (Brown, 2003). Este desorden neurobiológico o trastorno del desarrollo de la autorregulación es en la actualidad uno de los más estudiados y controversiales en cuanto se desconoce su etiología exacta habiendo quienes consideran que este trastorno en sí no tiene fundamento científico, sin embargo, en la práctica clínica hay evidencias basadas en los hallazgos anatómicos cerebrales, bioquímicos, neurofisiológicos y genéticos así como estudios en diversos campos como el realizado por R. A. Barkley (2009) cuyo resultado muestra que el 80\% de los casos de individuos diagnosticados con TDAH pueden ser de origen hereditario y el $20 \%$ puede tener otras etiologías, su diagnóstico aunque es clínico se basa es en los criterios establecidos en el Manual Diagnóstico y Estadístico de la Academia Americana de Psiquiatría DSM V (APA, 2013) y en la CIE 11 ( OMS, 2018) no ameritando generalmente estudios de laboratorio, exámenes neuropsicológicos ni de neuroimágenes.

Este trastorno tiene mayor prevalencia en los varones que en las niñas y a nivel mundial se estima que entre el 5 y $6 \%$ de la población infantil mundial posee este trastorno R. A. Barkley (2011) lo que lleva a pensar que es uno de los trastornos más comunes en edad infantil pudiendo este permanecer hasta la edad adulta por lo que estadísticamente se haya solo en Estados Unidos entre el 4 y $5 \%$ de la población adulta diagnosticada con este déficit R. A. Barkley (2011)

El término de hiperactividad se introdujo por primera vez en el DSM-II en 1968 y ha ido evolucionando según el síntoma al que se le haya dado mayor trascendencia en las investigaciones es así como (Bernabéu, 2014) en concordancia con el DSM V publicado por la APA (2013) destaca que las manifestaciones principales del TDAH son la falta de atención, la hiperactividad y la impulsividad, otros autores como (Barkley, 1995) subrayan como eje central las dificultades de autocontrol describiendo el trastorno como un déficit para autoregular la conducta no como una patología que dificulta el mantener la atención.

De acuerdo al DSM V se establecen 3 subtipos del TDAH: presentación combinada, presentación predominante con falta de atención y presentación predominante hiperactiva/impulsiva esta clasificación se establece teniendo en cuenta el síntoma más preponderante. En el DSM- IV revisión de este manual hecha en 1987 se agruparon las tres características fundamentales del síndrome para unificar el diagnóstico, pero fue con la revisión del DSM IV TR (APA, 1994/2000) que el trastorno pasa a llamarse TDAH, en la última versión el DSM V (2013) se introducen algunos cambios que afectan directamente el criterio diagnóstico. 
Por otra parte es importante señalar que entre el $60-80 \%$ de niños con hiperactividad infantil tenían problemas académicos importantes (Barkley, 1982), cabe resaltar que estas falencias son significativas en el área de lectura y escritura siendo algunas de estas el hecho de presentar frecuentes omisiones de palabras o letras, sustituciones de unas letras por otras, inversiones de letras asì mismo es importante destacar que es menor el nivel de comprensión lectora al enfrentarse a textos largos a pesar de no tener puntuaciones bajas en vocabulario. Otras dificultades que presentan los niños con el trastorno es la falta de comprensión en instrucciones escritas, por lo que generalmente un niño que realiza una tarea puede solo considerar algunas variables en su ejecución esto resultado de carecer de estrategias cognitivas que le permitan organizar la información, adaptarse a nuevas tareas y trabajar sin impulsividad, por otra parte Miranda, García y Jara (2001), Miranda et al (1998) indican que los niños realizan bien actividades de memoria cuando los materiales están altamente estructurados y no les exigen que elaboren estrategias de organización. Esto lo explica (García y Magaz, 2000) quien expone que la ausencia de atención sostenida, los cambios atencionales frecuentes y la escasa calidad atencional constituyen la principal causa de que no se adquieran hábitos y destrezas cognitivas, Ureña (2007) por otra parte expone que las funciones ejecutivas influyen en el comportamiento y en el rendimiento académico de los niños con TDAH los cuales tienen una ausencia o reducción de verbalizaciones internas, que es una de las más importantes estrategias para la regulación de la conducta.

\section{Metodología}

La investigación estuvo enmarcada dentro de un estudio correlacional porque se buscó conocer la relación de los movimientos sacádicos y de la comprensión lectora con el TDAH (Trastorno por déficit de atención e hiperactividad), se utilizó el método inductivo debido a que se tomó una muestra de niños cuyos resultados se empleó para extraer conclusiones de carácter general, la investigación se realizó desde un enfoque cualitativo porque describió los sujetos de estudio a partir de sus experiencias, se utilizó la observación y la experimentación.

La investigación se llevó a cabo en tres fases para su realización, en una primera fase, se realizó la recolección de datos llevándose a cabo entrevistas a padres de familias, docentes y estudiantes de la institución con y sin TDAH para conocer el grado de dificultad en la comprensión lectora y su funcionalidad visual en términos de movimientos oculares, se eligió la muestra de la población total y se aplicó la prueba King Devick y la prueba prolec -r a los 30 niños seleccionados, una segunda fase consistió en el análisis de la información en este caso se procesó los datos que se obtuvieron de las entrevistas semiestructuradas que se les realizó a 50 personas entre estudiantes, docentes y padres de familia y en la tercera fase de la investigación se realizó la presentación de los resultados a la comunidad académica.

La investigación busca relacionar las variables comprensión lectora, movimientos sacádicos y TDAH para lo cual se tomó una muestra que estuvo compuesta por 30 niños, 15 niños diagnosticados con TDAH y 15 sin este déficit en edades entre los 7 y 12 años eligiéndose este grupo de edad teniendo en cuenta que según la encuesta de salud mental realizado por el Ministerio de Salud ( 2017) este es el trastorno más frecuente en la niñez y la adolescencia, los niños se encontraban escolarizados, (de primero a quinto grado), algunos de los cuales presentaban dificultades en cuanto a la decodificación y reconocimiento de letras y palabras realizando omisiones, sustituciones, inversiones y regresiones y saltos de renglones se les aplicó las pruebas King Devick para evaluar los movimientos sacádicos y el test de PROLEC-R para valorar el nivel de comprensión lectora y al final se comparar los resultados.

En la aplicación de las pruebas se tomaron muestras de varios cursos de primero a quinto grado del colegio de jornadas de la mañana, se obtuvo el listado de los niños de cada curso de los registros de la oficina de psico orientación de la escuela que tenían el déficit y de los niños sin TDAH que presentaban dificultades en la comprensión lectora.

Para la evaluación de la comprensión lectora a los estudiantes de primaria con TDAH se utilizó la Prueba Prolec, esta prueba evalúa las pruebas se realizaron en un espacio tranquilo y sin distracciones bien iluminado y en general con condiciones adecuadas. evalúo los procesos que intervienen en la comprensión de material escrito y detectó las dificultades en la capacidad 
lectora de los niños, la prueba se realizó con una duración variable entre los 20 minutos y $40 \mathrm{mi}-$ nutos. La batería se compone de nueve pruebas: Nombre o sonido de las letras, igual-diferente, lectura de palabras, lectura de pseudopalabras, estructuras gramaticales, signos de puntuación, comprensión de oraciones, comprensión de textos y comprensión oral.

- Procesos iniciales de identificación de letras: consta de dos tareas que son: nombre o sonido de las letras e Igual-Diferente.

- Procesos léxicos: la prueba consta de una lista de palabras y otra lista de pseudopalabras

- Procesos gramaticales: esta prueba está compuesta por las tareas de estructuras gramaticales y signos de puntuación.

- Procesos semánticos: para la valoración de los procesos semánticos esta prueba establece tres tipos de tareas: comprensión de oraciones, comprensión de textos y comprensión oral.

La otra prueba realizada a la muestra fue el test de King-Deving (K-D) el cual consiste en leer tres cartas con números que los estudiantes tenían que leer lo más rápido posible sin fallos, con esto se midió la velocidad lectora y si los movimientos sacádicos los realizaban de forma adecuada, si tenían regresiones y si saltaban líneas. Con esta prueba de seguimiento ocular se pudo comprobar si los niños realizaban los movimientos oculares de forma adecuada, cuál era su amplitud de visión, si era capaz de realizar los seguimientos oculares sin mover la cabeza, etc. Para su realización se les explicó a los niños en qué consistía y como debían realizarla siguiendo los números señalados sin mover la cabeza, es decir, la lectura tenía que ser únicamente ocular. Esta prueba consistió en una carta de demostración no evaluable y tres subtest que constan de 40 números distribuidos en ocho líneas organizados de diferentes formas en el espacio.

Se inició con la carta de demostración para comprobar si se habían comprendido las instrucciones, una vez comprobado esto se continuó con las tarjetas:

Carta I: esta tarjeta consta de números colocados horizontalmente por líneas sin flechas.

Carta II: en esta segunda carta no hay líneas que unan los números

Carta III: en estas tarjetas no hay líneas y los números están más juntos.
Se cronometrizó el tiempo y se anotaron los errores que cometió el niño al leer un texto corto tomado del manual de la prueba prolec-r el cual tiene como nombre "el ratel", este tenía que ser leído por el niño tomándosele el número de palabras que tardaba en leer en un minuto y sin que este moviera la cabeza.

\section{Resultado}

En este apartado se describen los resultados obtenidos después de analizar las pruebas Prolec- r y King Devick. Para realizar el análisis de la estadística descriptiva se utilizó Microsoft Excel y para obtener la estadística correlacional se empleó el programa estadístico spss. La estadística descriptiva se halló a través de Microsoft Excel, obteniéndose el porcentaje y la frecuencia de niños con TDAH y sin TDAH que tuvieron una valoración normal, moderada o severa dependiendo de los resultados en las diferentes sub pruebas del test de prolec-r y los porcentajes de niños con TDAH y sin TDAH que tuvieron o no movimiento de cabeza en la prueba King Devick, Por otro lado para la estadística correlacional se aplicó específicamente el coeficiente de Spearman para hallar la relación entre las dos variables movimientos sacádicos y la comprensión lectora, es una medida correlación, asociación o interdependencia entre dos variables aleatorias que oscila entre -1 y +1 indicándonos asociaciones negativas o positivas respectivamente, el o cero, significa no correlación pero no independencia asimismo se utilizó el Mann-Whitney para hallar la heterogeneidad de las muestras al realizarse la observación de forma independiente en cuanto a comprensión lectora y movimientos de cabeza.

Teniendo en cuenta los resultados hallados en la estadística descriptiva se puede afirmar que no existen diferencias significativas en el análisis de los resultados de la prueba Prolec-r y King Devick de los dos grupos de niños evaluados (con y sin TDAH). La prueba Prolec-r midió la frecuencia de niños con TDAH y sin TDAH que tuvieron una valoración normal, moderada o severa en las diferentes sub pruebas del test, de igual manera la estadística descriptiva no mostró diferencias significativas en los porcentajes de niños con TDAH y sin TDAH que tuvieron o no movimiento de cabeza en la prueba King Devick. Cabe resaltar que a través de la observación realizada se puede afirmar que la comprensión lectora de los niños con TDAH se ve afectada por 
diversos factores entre los cuales podemos citar el hecho de si el niño está o no medicado, la edad y el grado de escolarización, los niños con TDAH presentaron mayor dificultad al leer textos utilizando los signos de puntuación y al hacer las entonaciones, un mayor problema a la hora de leer pasajes largos y al realizar inferencias acerca de los textos pues tendían a cansarse con mayor facilidad, llegando algunas veces a inventar las respuestas cuando se les preguntaba acerca del texto, por otra parte los niños que no presentan el déficit a la hora de responder daban más detalles acerca del texto, es preciso señalar que la mayoría de los niños presentan movimientos de cabeza y dificultades como saltar renglón, ubicar el texto con el dedo, lectura lenta silábica y con regresiones y omisiones de letras u palabras, inversiones y confusiones en las letras como la g y j q y k l y r entre otras y combinaciones de consonantes como pr, br, cl.

\section{EsTADÍSTICA DESCRIPTIVA}

Tabla 1. RESULTADO PRUEBA PROLEC-R

\begin{tabular}{|c|c|c|c|c|c|c|c|}
\hline \multicolumn{8}{|c|}{ TABLA DE RESULTADOS PROLEC-R } \\
\hline \multirow{10}{*}{$\begin{array}{c}\text { NINOOS SIN } \\
\text { TDH }\end{array}$} & INDICE PRINCIPALES & SEVERO & MODERADO & NORMMAL & SEVERO \% & MODERADO $\%$ & NORMAL \% \\
\hline & COMPRENSION DE ORACIONES & 5 & 4. & 6 & $33 \%$ & $27 \%$ & $40 \%$ \\
\hline & COMPRENSION DE TEXTOS & 4 & 4. & 6 & $27 \%$ & $27 \%$ & $40 \%$ \\
\hline & COMPRENSION ORAL & 1 & 4. & 8 & $7 \%$ & $27 \%$ & $53 \%$ \\
\hline & ESTRUCTURAS GRAMATICALES & 4 & 3. & 9 & $27 \%$ & $20 \%$ & $60 \% 5$ \\
\hline & IGUAL-DIFERENTE & 1 & 12 & 3 & $7 \%$ & $80 \%$ & $20 \%$ \\
\hline & LECTUFA DE PALABRAS & 4 & 7 & 4 & $27 \%$ & $47 \%$ & $27 \%$ \\
\hline & LECTURA DE PSEUDOPALABRAS & 6 & 8. & 1 & 4056 & $53 \%$ & $7 \%$ \\
\hline & NOMBRE DE LETRAS & 10 & 3. & 2 & $67 \%$ & $20 \%$ & $13 \%$ \\
\hline & SIGNOS DE PUNTUACION & 10 & 2. & 1 & $67 \%$ & $13 \%$ & $7 \%$ \\
\hline \multirow{10}{*}{$\begin{array}{c}\text { NIÑOS CON } \\
\text { TDH }\end{array}$} & INDICE PRINCIPAL & SEVERO & MODERADO & NOFMAL & SEVERO $\%$ & MODERADO $\%$ & NORMAL \% \\
\hline & COMPRENSION DE ORACIONES & 2 & 3. & 9 & 1356 & $20 \%$ & $60 \%$ \\
\hline & COMPRENSION DE TEXTOS & 1 & 2 & 11 & $7 \%$ & $13 \%$ & $73 \%$ \\
\hline & COMPRENSION ORAL. & 0 & 2. & 12 & 056 & $13 \%$ & $80 \% 6$ \\
\hline & ESTRUCTURAS GRAMATICALES & 2. & 3. & 10. & $13 \%$ & $20 \%$ & $67 \%$ \\
\hline & IGUAL-DIFERENTE & 4 & 11 & 0 & $27 \%$ & $73 \%$ & $0 \%$ \\
\hline & LECTURA DE PALABRAS & 5 & 7 & 3 & 3355 & $47 \%$ & $20 \%$ \\
\hline & LECTURA DE PSEUDOPALABRAS & 6 & 4. & 5 & $40 \%$ & $27 \%$ & $33 \%$ \\
\hline & NOMBRE DE LETRAS & 2 & 6 & 7 & 1356 & $40 \%$ & $47 \%$ \\
\hline & SIGNOS DE PUNTUACION & 13 & 2 & c & $87 \%$ & $13 \%$ & $0 \%$ \\
\hline
\end{tabular}

Tabla 2.

TABLA DE RESULTADO TEST K-D (SIN TDH)

\begin{tabular}{|c|c|c|c|c|c|c|c|}
\hline MUESTRA & Edad & $\begin{array}{c}\text { Nómcro de } \\
\text { palabras } \\
\text { Icidas por } \\
\text { minuto }\end{array}$ & $\begin{array}{c}\text { Tlempo } \\
\text { total en } \\
\text { Icer las tues } \\
\text { tarjetas }\end{array}$ & $\begin{array}{c}\text { NE de } \\
\text { errores } \\
\text { totales en } \\
\text { las tres } \\
\text { tarjetas }\end{array}$ & $\begin{array}{c}\text { Movimient } \\
\text { ode la } \\
\text { cabera } \\
\text { (si/no) }\end{array}$ & $\begin{array}{c}\text { NINOS CON } \\
\text { MOVIMIENT } \\
\text { OS DE } \\
\text { CABEZA }\end{array}$ & $\begin{array}{c}\text { MIÑOS SIN } \\
\text { MOVIMIEN } \\
\text { TOSDE } \\
\text { CABEZA }\end{array}$ \\
\hline 2 & 9 & $\hat{8}$ & $14424 g$ & 0 & si & $73 \%$ & $27 \%$ \\
\hline 2 & 7 & 10 & $21 / s=1$ & $v$ & si & & \\
\hline 3 & 10 & 32 & $1 / 4 \circ 5$ & 4 & ei & & \\
\hline 4 & 7 & 68 & $100=$ tely & 0 & si & & \\
\hline 5 & 7 & 34 & Hy $3 \mathrm{SO}_{5}$ & 1 & i & & \\
\hline 6 & 7 & $6: 2$ & $21 ;>8=E$ & 5 & 20 & & \\
\hline 8 & 8 & $6: 2$ & $1108=y$ & 2 & =i & & \\
\hline 8 & 10 & 44 & $\gamma$ asy & 3 & 100 & & \\
\hline 9 & 3 & 36 & $C, 2 \mathrm{tg}$ & 2 & =i & & \\
\hline 10 & 8 & 48 & $21: 2 x g$ & $\because$ & 20 & & \\
\hline 12 & 9 & 8 & $4 j 3 x_{0}$ & 3 & ai & & \\
\hline 12 & 9 & $3 y$ & $B_{3} ; x_{5}$ & 2 & si & & \\
\hline 13 & 9 & 36 & $2 \geq 5,3=1$ & $: 2$ & 20 & & \\
\hline 14 & 9 & 64 & $79 \mathrm{scg}$ & 1 & 3i & & \\
\hline 15 & I] & 38 & $7>$ ieg & 2 & ง & & \\
\hline
\end{tabular}


Tabla 3.

TABLA DE RESULTADO TEST K-D (CON TDH)

\begin{tabular}{|c|c|c|c|c|c|c|c|}
\hline MUESTRA & Edad & $\begin{array}{c}\text { Nùmero de } \\
\text { palabras } \\
\text { leidas por } \\
\text { minuto }\end{array}$ & $\begin{array}{c}\text { Tiempo } \\
\text { total en } \\
\text { leer las tres } \\
\text { tarjetas }\end{array}$ & $\begin{array}{c}\text { Ni de } \\
\text { errores } \\
\text { totales en } \\
\text { las tres } \\
\text { tarjetas }\end{array}$ & $\begin{array}{c}\text { Movimient } \\
\text { o de la } \\
\text { cabeza } \\
\text { (sí/no) }\end{array}$ & $\begin{array}{c}\text { NINOS CON } \\
\text { MOVIMIEN } \\
\text { TOS DE } \\
\text { CABEZA }\end{array}$ & $\begin{array}{c}\text { NIÑOS SIN } \\
\text { MOVIMIEN } \\
\text { TOS DE } \\
\text { CABEZA }\end{array}$ \\
\hline 16 & 8 & 20 & $75 \operatorname{seg}$ & & ถู & $73 \%$ & 278 \\
\hline 17 & 9 & 33 & $72 \operatorname{seg}$ & 4 & si & & \\
\hline 18 & 8 & 40 & $74 \mathrm{seg}$ & 1 & si & & \\
\hline 19 & 8 & 37 & $76 \operatorname{seg}$ & 3. & s่ & & \\
\hline 20 & 12 & 64 & 94 seg & & no & & \\
\hline 21 & 9 & 19 & $79 \mathrm{seg}$ & 4 & no & & \\
\hline 22 & 8 & 60 & $68 \mathrm{seg}$ & 4 & si & & \\
\hline 23 & 8 & 43 & 70 seg & 1 & si & & \\
\hline 24. & 8 & 44. & $52 \operatorname{seg}$ & 2 & si & & \\
\hline 25 & 8 & 78 & $74 \mathrm{seg}$ & 1 & no & & \\
\hline 26 & 8 & 69 & $72 \mathrm{seg}$ & 2 & si & & \\
\hline 27 & 11 & 69. & $52 \operatorname{seg}$ & 3 & si & & \\
\hline 28 & 12 & 36 & $73 \operatorname{seg}$ & 4 & si & & \\
\hline 29 & 11 & 66 & $56 \mathrm{seg}$ & 1 & no & & \\
\hline 30 & 12 & 51 & $67 \mathrm{seg}$ & 3 & si & & \\
\hline
\end{tabular}

\section{ESTADISTICA CORRELACIONAL}

En este estudio se buscó conocer si existía relación entre las dos variables: comprensión lectora y los movimientos oculares

SPEARMAN

NONPAR CORR

/VARIABLES=COM_TEX MOV_CABEZ

Se empleó el coeficiente correlacional de Spearman arrojando los siguientes resultados:

/PRINT=SPEARMAN TWOTAIL NOSIG $/$ MISSING $=$ PAIRWISE

\section{Nonparametric Correlations}

Tabla 4. Coeficiente correlacional de Spearman comprensión lectora- movimientos sacádicos

Correlations

\begin{tabular}{|c|c|c|c|c|}
\hline & & & $\begin{array}{l}\text { COMPRESSIO } \\
\text { N DE TEXTOS }\end{array}$ & $\begin{array}{l}\text { MOVIMIENTO } \\
\text { DE LA CABEZ }\end{array}$ \\
\hline \multirow[t]{2}{*}{ Spearman's rho } & $\begin{array}{l}\text { COMPRESSION DE } \\
\text { TEXTOS }\end{array}$ & $\begin{array}{l}\text { Correlation Coefficient } \\
\text { Sig. (2-tailed) } \\
\text { N }\end{array}$ & $\begin{array}{r}1,000 \\
30\end{array}$ & $\begin{array}{r}, 044 \\
, 818 \\
30\end{array}$ \\
\hline & $\begin{array}{l}\text { MOVIMIENTO DE LA } \\
\text { CABEZ }\end{array}$ & $\begin{array}{l}\text { Correlation Coefficient } \\
\text { Sig. (2-tailed) } \\
\text { N }\end{array}$ & $\begin{array}{r}.044 \\
.818 \\
30\end{array}$ & $\begin{array}{r}1,000 \\
30\end{array}$ \\
\hline
\end{tabular}


El Sig. (2-tailed) indica un coeficiente de 0.818 el cual es superior a 0,05 por lo cual no se puede demostrar que haya una relación significativa entre las dos variables movimientos sacádicos y comprensión de lectura. NPAR TESTS

/M-W= COM_TEX BY TDH (1 2)

/MISSING ANALYSIS.

Según la prueba paramétrica Mann Whitney el Sig. (2-tailed) indica un coeficiente de 0.74 superior al 0,05 por lo tanto no se puede demostrar una diferencia significativa entre el valor de los grupos de niños sin TDAH y de niños con TDAH en cuanto a la comprensión de lectura.

Para movimientos de cabeza durante la prueba K-D el sig. 2 tailed arroja un coeficiente de uno $(1,000)$ el cual es superior a 0,05 por lo tanto no se puede establecer una diferencia significativa entre el grupo de niños con TDAH y el grupo de niños sin TDAH en cuanto a movimientos de cabeza.

Las pruebas empleadas prolec-r y King Devick nos permitieron evaluar a los niños en las diferentes variables relacionadas, la prueba prolec-r es una prueba diagnóstica que evalúa los procesos que intervienen en la comprensión escrita y detecta las dificultades lectoras. El prolec-r nos proporciona información sobre cuáles son los procesos cognitivos responsables de las dificultades lectoras.

En la prueba prolec en la subprueba nombre de letras los niños sin TDAH presentan una frecuencia de severo $67 \%$, moderado $20 \%$ y normal $13 \%$ el porcentaje de niños con TDAH es $13 \%$ severo, moderado $40 \%$ y normal $47 \%$ el objetivo de esta prueba es comprobar si el niño conoce todas las letras y su pronunciación, ya que este es un paso fundamental para poder leer

igual -diferente tiene como objetivo esta tarea tiene como objetivo conocer si el niño es capaz de segmentar e identificar las letras que componen cada palabra que tiene que leer, o por el contrario, realiza una lectura logográfica. Para ello se le proporcionan pares de palabras y pseudopalabras (p. ej., caballo-cabalo) iguales o diferentes y será el niño quien tenga que indicarlo de esta es correctamente se puede observar que el porcentaje de niños sin TDAH que presenta una frecuencia de severa, es el $7 \%$, moderada $80 \%$ y normal $20 \%$ y el porcentaje de niños con
TDAH que presenta una frecuencia severa es $27 \%$, moderada $73 \%$ y normal $\%$, en la subprueba de comprensión de oraciones el porcentaje de niños sin TDAH es severo 33\%, moderado $27 \%$ y normal 40\% con TDAH es $13 \%$ severo, $20 \%$ moderado y $60 \%$ normal, en comprensión de textos los niños sin TDAH presentan un porcentaje de severo $27 \%$, moderado $27 \%$ y $40 \%$ normal los niños con TDAH presentan un porcentaje de severo7\%, $13 \%$ moderado y normal $73 \%$, comprensión oral niños sin TDAH severo 7\%, 27\% moderado y $53 \%$ normal los niños con TDAH presentan $0 \%$, severo, $13 \%$, moderado y $80 \%$ normal, en la subprueba de procesos gramaticales los niños sin TDAH presentan un porcentaje de $27 \%$ severo, $20 \%$ moderado y normal $60 \%$, los niños con TDAH por su parte presentan un porcentaje de $13 \%$ severo, $20 \%$ moderado y $67 \%$ normal, en la subprueba de lectura de palabra los niños sin TDAH obtuvieron un porcentaje de $27 \%$ severo, $47 \%$ moderado y $27 \%$ normal, los niños con TDAH obtuvieron un porcentaje de $33 \%$ severo, $47 \%$ moderado y normal $20 \%$ en otra subprueba denominada lectura de pseudopalabras los niños sin TDAH obtuvieron un porcentaje de $40 \%$ severo, $53 \%$ moderado y $7 \%$ normal, los niños sin TDAH un porcentaje de $40 \%$ severo, $27 \%$ moderado y $33 \%$ normal, por último en la prueba de signos de puntuación los niños con TDAH presentaron un porcentaje de $67 \%$ severo, $13 \%$ moderado y $7 \%$ normal en comparación con los niños con TDAH que presentaron un porcentaje de severo $87 \%, 13 \%$ moderado, y o\% normal.

La prueba de K-D de los niños sin TDAH arroja un porcentaje de $73 \%$ de niños con movimiento de cabeza al leer el texto y $27 \%$ de niños de no presentan movimientos de cabeza al leer el texto, esto en comparación con los niños con TDAH quienes presentan un porcentaje igual a los niños sin TDAH con un porcentaje de $73 \%$ de niños con movimiento de cabeza al leer el texto y $27 \%$ de niños de no presentan movimientos de cabeza al leer el texto. Estas pruebas evalúan las problemáticas de comprensión de textos antes mencionadas y los movimientos oculares de los niños con TDAH y sin TDAH.

\section{Conclusión}

Alrededor de un 50\% de niños con TDAH tiene problemas en el aprendizaje de la lectura, de la escritura o de las matemáticas que inciden 
negativamente en el rendimiento académico y en el nivel de educación que alcanzan. Pero, en comparación con el cálculo o con el acceso léxico, la comprensión lectora de los niños con TDAH ha suscitado poco interés entre los investigadores. La investigación corroboró lo que algunos estudios como los de (Berthiaume, Lorch y Milich, 2009) han señalado: que los niños TDAH realizan un menor número de inferencias y tienen más problemas para reconocer las incoherencias de un texto. No se evidencian problemas significativos en los movimientos sacádicos y en la comprensión lectora de los niños con TDAH en comparación con el grupo control (niños que no presentan este déficit) así mismo, las pruebas realizadas, aunque no relacionar el TDAH con las variables movimientos oculares y comprensión de lectura estadísticamente permiten observar problemas en los procesos neuropsicológicos como atención, memoria etc.

$\mathrm{Al}$ analizar la prueba de comprensión lectora se encontraron falencias en algunos niños por lo cual es importante llevar a cabo un programa de intervención con el propósito de mejorar los diferentes niveles de comprensión lectora, por lo cual se plantearon actividades que permitieran su progreso, que no les ocasionara cansancio y que los motivara, por lo que se desarrollaron sesiones de 30 a 40 minutos, de forma lúdica en un espacio donde no existieron distracciones.

Después de conocer los aspectos que deben mejorarse en cuanto a movimientos sacádicos se aplicó un programa de intervención durante 3 meses con una serie de actividades para realizar de forma lúdica en un ambiente tranquilo y sin distracciones. Cada sesión tuvo una duración de unos 40 minutos en el cual se desarrollaron de forma alternada ejercicios de movilidad ocular, así como ejercicios de movimientos sacádicos.

Los resultados de esta investigación confirman lo expresado en estudios internacionales y nacionales en cuanto a la relación poco significativa entre las variables comprensión lectora, movimientos sacádicos y TDAH, analiza algunos aspectos que corresponden a la comprensión de lectura.

Es necesario indagar más esta área de comprensión lectora con el fin de brindar una mejor ayuda a las necesidades específicas de los niños con TDAH 


\section{Referencias bibliográficas}

American Psychiatric Association (2014). Manual Diagnóstico y Estadístico de los Trastornos Mentales (DSM-5), $5^{\mathrm{a}}$ Ed. Madrid: Editorial Médica Panamericana.

American Psychiatric Association. (1995). Manual Diagnóstico y Estadístico de los Trastornos Mentales: DSM-IV. Barcelona: Masson S.A.

De la Torre Alcalá, A. (2.001). Ejercicios de coordinación visomotora. Editorial CEPE. Madrid.

De la Torre Alcalá, A. (2006). Ejercicios perceptivos motores de orientación espacial. Editorial CEPE. Madrid.

Del Campo Adrián, M. (2002). Dificultades de aprendizaje, e intervención psicoeducativa. Sanz y Torres. Madrid.

Departamento de educación del gobierno de Navarra (servicio de igualdad de oportunidades, participación educativa y atención al profesorado. CREENA). (2012), Entender y atender al alumnado con déficit de atención e hiperactividad (TDAH) en las aulas. España, Navarra: Fondo de Publicaciones del Gobierno de Navarra.

Fernández Baroja, F. (2.006). La dislexia. Origen diagnóstico y recuperación. Editorial CEPE. Madrid.

Fernández Trespalacios, J.L. (1.992). Psicología General II. Universidad Nacional de Educación a Distancia. Madrid.

García Madruga, J. y Gutiérrez Martínez, F. (2.002). Psicología Evolutiva. Desarrollo cognitivo y lingüístico. Universidad Nacional de Educación a Distancia. Madrid.

Gratch, L. (2009). El Trastorno por déficit de atención (ADD-ADHD). Buenos Aires: Panamericana.

Ospina Aroca, L. (2014). Influencia de los movimientos sacádicos y comprensión lectora en el rendimiento académico. Universidad Internacional de la Rioja (dissertation master). Recuperado el 17 de agosto de 2018 de 2018 de http:// reunir.unir.net/bitstream/handle/123456789/2398/ospina\%20aroca.pdf?sequence=1

Álvarez, D. (2014). Incidencia de los movimientos sacádicos en la velocidad y comprensión lectora en alumnos de primaria. Universidad Internacional de la Rioja. (dissertation master). Recuperado el 23 de agosto de 2018 de http://reunir.unir. net/bitstream/handle/123456789/2838/alvarez\%2omauleon.pdf?sequence=1.

Begoña, R.B. (2015). Detección e intervención del TDAH en la educación. Universidad Internacional de la Rioja. (dissertation master). recuperado el 13 de septiembre de 2018 de http://reunir.unir.net/bitstream/handle/123456789/2852/ Bego\%C3\%B1a_Ripol_Baixas.pdf?sequence=1

Barrachina, L. (2015) La comprensión lectora, la memoria de trabajo los movimientos oculares y la discriminación auditiva. Universidad Internacional de la Rioja. (dissertation master). Recuperado el día 29 de agosto de 2018 de http://reunir. unir.net/bitstream/handle/123456789/3425/BARRACHINA\%2oMOR\%2c\%2oLAIA.pdf?sequence=1

Soutullo, C. (2007). Comorbilidad y diagnóstico diferencial. Recuperado el 17 de septiembre de 2018 de http://www.apalweb. org/docs/da4.pdf.

Egea V. (2012). Ejercicios de comprensión lectora. Edunoi centro psicopedagógico. Alicante. Recuperado el 31 de agosto de 2018 de http://www.edunoi.com/ejercicios-de-comprension-y-razonamiento/

Rief, S.F. (1999). Cómo tratar y enseñar al niño con problemas de atención e hiperactividad: Técnicas, estrategias e intervención para el tratamiento del TDA / TDAH. Buenos Aires: Paidós.

Vallés, A. (1998a). Las dificultades de aprendizaje por déficits de la atención. Dificultades de aprendizaje e intervención psicopedagógica (pp. 395-433) Valencia: Promolibro.

Grupo de trabajo de la GPC de TDAH. (2010). Guía de práctica clínica en el SNS trastorno de déficit por atención TDAH. Cataluña: Ministerio de sanidad política social y sanidad.

Sánchez, R. (2015). Aplicación de la prueba visual de seguimientos oculares para la lectura King Devick. Universidad Internacional de la Rioja. (dissertation master). Recuperado el 19 de noviembre de 2018 de https://es.calameo.com/ read/o01001281388e7fa978e6.

Arbieto, Kenny. (2002). Trastorno por déficit de atención. Recuperado el 12 de septiembre de 2018 de http://www. psicopedagogia.com/trastorno-por-deficit-de-atencion

Esteban, V. (2013). Tratamiento de un caso por déficit de atención e hiperactividad recuperado el 12 de septiembre de 2018. Tomado de http://www.psicoterapeutas.com/paginaspersonales/Violeta/tdah_caso.html de 
Pérez, V. (2016). ¿Es correcto diferenciar entre TDA y TDAH como si fueran dos trastornos independientes? Recuperado el 19 de noviembre de 2018 de http://www.tdahytu.es/tda-tdah-sin-hiperactividad/

Lora Espinosa A, Díaz Aguilar MJ. Abordaje del trastorno por déficit de atención con/sin hiperactividad desde la visión del pediatra de cabecera. Rev Pediatr Aten Primaria Supl. 2011;(20):115-26.

Martín Lobo, P (2.003). La Lectura. Procesos neuropsicológicos de aprendizaje, dificultades, programas de intervención y estudio de casos. Lebón. Barcelona

Miranda-Casas A, Fernández MI, Robledo P, García-Castellar R. Comprensión de textos de estudiantes con trastorno por déficit de atención/ hiperactividad: ¿̇qué papel desempeñan las funciones ejecutivas? Rev Neurol 2010; 50 (Supl 3): S135-42.

Ripoll, J. C. (2014). Estudiar y hacer la tarea. Alumnos de la ESO incluso con TDAH. Madrid: CEPE.

Ripoll, J. C. Hualde, M. (2009). Alumnos distraídos, inquietos e impulsivos (TDA-H). estrategias para atender su tratamiento educativo en educación primaria. Madrid: CEPE.

Morchòn, L. (2011) Eficacia de un programa de intervención con terapia visual en la escuela. Universidad Internacional de la Rioja (dissertation master). Recuperado el 29 de septiembre del 2018 de http://upcommons.upc.edu/bitstream/ handle/2099.1/13817/TFM\%20Lucia\%20Morch\%C3\%B3n.pdf

Russo, N. (2006). Niños desatentos e hiperactivos. Recuperado el 15 de septiembre de 2018 de https://www.psicopedagogia. com/articulos/?articulo=669

Fuentes, L. (2009). Diagnóstico de comprensión lectora en educación básica en Villarrica y Loncoche, Chile. Perfiles educativos, 31(125), 23-37. Recuperado el 13 de septiembre de 2018, de http://www.scielo.org.mx/scielo.php?script=sci_ arttext\&pid=So185-26982009000300003\&lng=es\&tlng=es

González, J. (2010). Cómo afrontar los problemas del TDAH en el área de lectura Recuperado el 16 septiembre de https:// tdahsalamanca.wordpress.com/2010/o6/30/como-afrontar-los-problemas-del-tdah-en-el-area-de-lectura/

Arbieto, K. (2002). Trastorno por déficit de atención. Recuperado el 14 de septiembre de 2018 de http://www. psicopedagogia.com/trastorno-por-deficit-de-atencion

Ripoll, J. C. (2018). Tratamiento educativo del TDAH. Recuperado el 19 de noviembre de 2018 de https://educaciontdah. wordpress.com/

Santos, C.\& Del Campo M. (2013) la incidencia del nistagmus en la eficiencia lectora de alumnos con baja visión. International Journal of Developmental and Educational Psychology. Recuperado el 19 de noviembre de 2018 de http:// www.redalyc.org/articulo.oa?id=349852058055

Monroy, J. \& Gómez, B. (2009). Comprensión lectora. Revista Mexicana de Orientación Educativa, 6(16), 37-42. Recuperado em 20 de noviembre de 2018, de http://pepsic.bvsalud.org/scielo.php?script=sci_arttext\&pid=S1665$75272009000100008 \& \operatorname{lng}=$ pt\&tlng=es.

Pardo, M. (2009). Disfunciones visuoperceptivas, oculomotoras, acomodativas y binoculares en niños con déficit de atención e hiperactividad. Universidad Politécnica de Cataluña. (Dissertation master). Recuperado el 19 de noviembre de 2018 de https://upcommons.upc.edu/bitstream/handle/2099.1/7876/TFMM\%C2\%AA\%20Carmen\%20Pardo.pdf

Inostroza, F. Estudiantes con déficit atencional enfrentando tareas de comprensión lectora y producción de textos: Un estudio comparativo en alumnado de $4^{\circ}$ año de educación primaria en Chile http://www.redalyc.org/ jatsRepo/1941/194154512006/html/index.html 
Revista incluida en los siguientes agregadores de contenidos
Revista incluida en los siguientes directorios

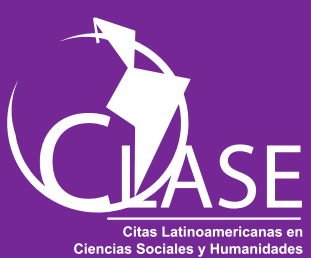

llboblat

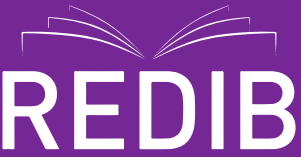

Red Iberoamericana

de Innovación y Conocimiento Cientifico

\section{Google Académico}
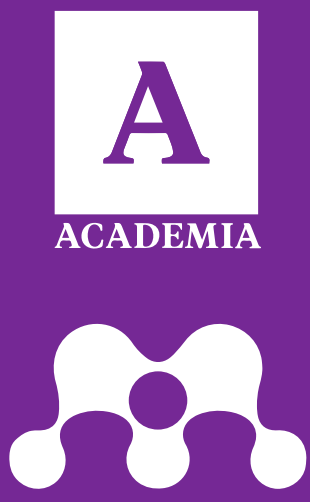

MENDELEY

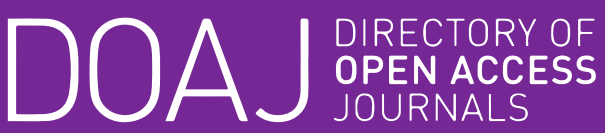

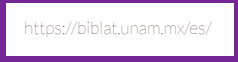
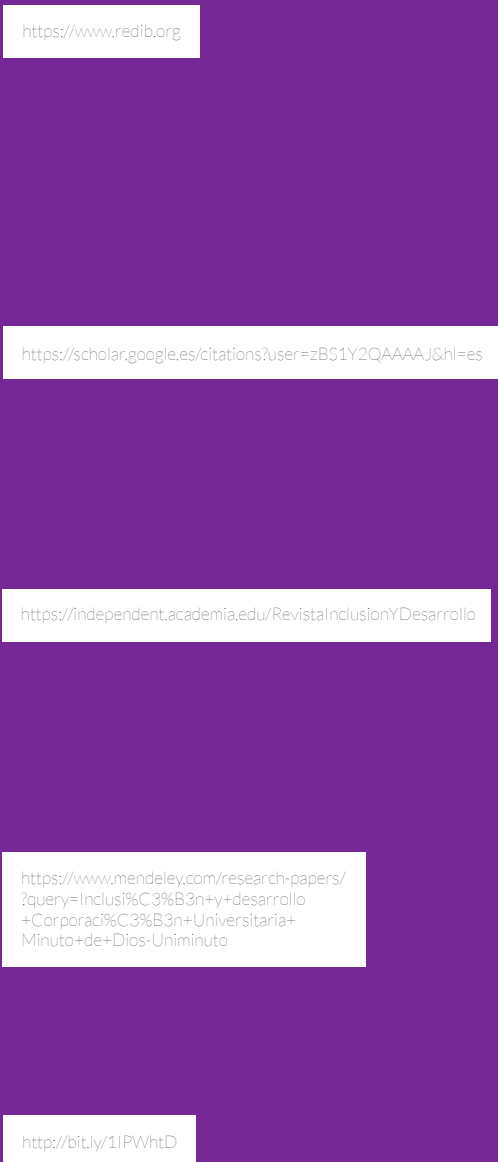

Revista incluida en la siguiente red social

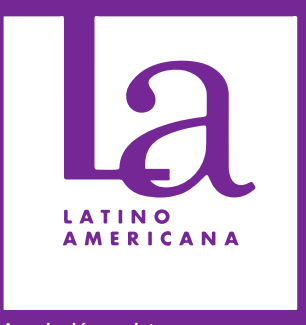



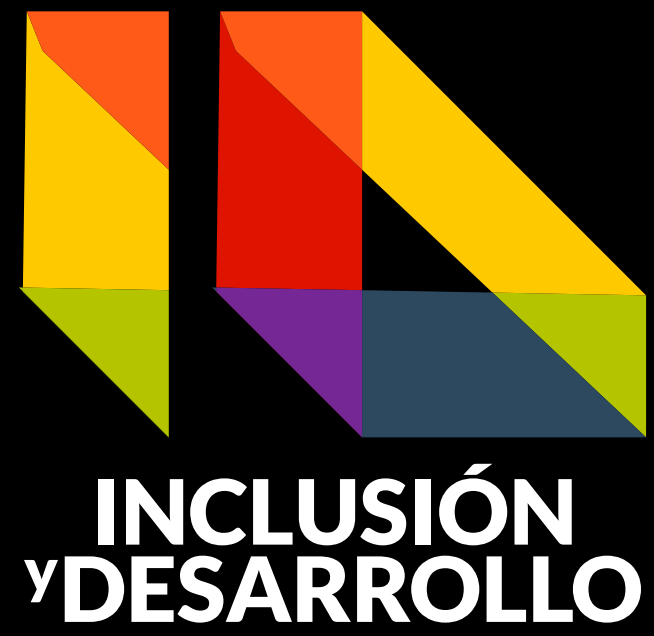

No. 1 Vol. 6 Año 2019 ISSN En línea: 2590-7700

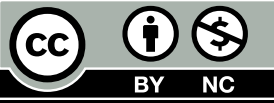

